\title{
GAUGE FUNCTIONS AND LIMIT SETS FOR NONAUTONOMOUS ORDINARY DIFFERENTIAL EQUATIONS
}

\author{
H. K. WILSON
}

\begin{abstract}
A gauge function $V$ for the differential equation (S) $x^{\prime}=f(x, t)$ is a scalar-valued function sufficiently smooth for $d V(\phi(t), t) / d t$ to exist almost everywhere for solutions $x=\phi(t)$, $t_{0} \leqq t<\tau_{\phi}^{+}$, of (S). Let (S) have gauge function $V$ that satisfies the following conditions: (1) $\lim _{t \rightarrow+\infty} V(x, t) \equiv \lambda(x)$ exists; (2) $V$ is continuous in $x$ uniformly with respect to $t$; (3) the upper, right derivate of $V$ with respect to (S) is nonpositive. Then, if a solution $x=\phi(t)$ of (S) has an $\omega$-limit point $p$, there is a unique constant $c(\phi)$ such that $\lambda(p)=c(\phi)$. An application to second order, linear equations is given.
\end{abstract}

1. Context of the discussion. Let $f$ be continuous on a cylinder $D=$ $P \times I$, where $P$ is a domain in $R^{n}$ and $I$ is an interval $[\beta,+\infty)$, and consider the differential equation

$$
x^{\prime}=f(x, t) .
$$

The fundamental qualitative problem for (S) is the description of the asymptotic behavior of its solutions. In this note, there is developed a technique for such description by means of gauge functions.

Let $Q \subset P$ be open. A gauge function for (S) is a function $V: Q \times I \rightarrow R^{1}$ that is bounded below on every cylinder $K \times I$ with compact base $K \subset Q$ and satisfies the following condition: For every compact neighborhood $K \times[a, b]$ in $Q \times I$, there is a number $L>0$ and an absolutely continuous function $\psi$ such that

$|V(x, t)-V(y, t)| \leqq L \cdot|x-y| \quad$ and $\quad|V(x, s)-V(x, t)| \leqq|\psi(s)-\psi(t)|$

for all $x, y \in K$ and $s, t \in[a, b]$. The derivative $D V$ of a gauge function $V$ with respect to the equation $(\mathrm{S})$ is defined by the relation

$$
D V(x, t)=\limsup _{h \rightarrow 0+} \frac{V(x+h f(x, t), t+h)-V(x, t)}{h} .
$$

Received by the editors January 25, 1972.

AMS 1970 subject classifications. Primary 34C99; Secondary 34A30, 34C05, 34D20.

Key words and phrases. Gauge function, $\omega$-limit set, positive limit set, asymptotic behavior of solutions, linear second order equation.

c. American Mathematical Society 1972 
Throughout the rest of the paper, consider as given a solution $x=\phi(t)$ of (S) that initiates at a point $\left(x_{0}, t_{0}\right)$ in $D$, has escape time $\tau_{\phi}^{+}$from $P$, and has at least one $\omega$-limit point in $P$. Further, suppose that $\phi(t)$ is in a subset $G$ of $P$ for $t_{0} \leqq t<\tau_{\phi}^{+}$and that $V: Q \times I \rightarrow R^{1}$ is a gauge function for (S) with $Q \supset \bar{G} \cap P$.

Below, we establish circumstances under which the $\omega$-limit set $\Omega_{\phi}$ of $\phi$ is a subset of the locus of precisely one equation

$$
\lim _{t \rightarrow+\infty} V(x, t) \equiv \text { constant. }
$$

Then, we illustrate the applicability of the results by solving a stability problem for a linear oscillator with one degree of freedom.

2. The results. It is a routine matter to establish that $V(\phi(t), t)$ is locally absolutely continuous. Consequently, $d V(\phi(t), t) / d t$ exists for almost all $t \in\left[t_{0}, \tau_{\phi}^{+}\right)$and equals $D V(\phi(t), t)$ when it does exist. Further, $V(\phi(t), t)$ is nonincreasing if $D V(\phi(t), t) \leqq 0$ for almost all $t$.

THEOREM 1. Suppose that $A$ is a nonempty subset of $Q$ such that $D V(x, t) \leqq 0$ for $(x, t)$ in the cylinder $A \times I$ and let the measure of $\left\{t: t \in\left[t_{0},+\infty\right)\right.$ and $\left.\phi(t) \notin A\right\}$ be zero. Then there is a number $v_{\phi}$ with the following property: If $\left\{t_{k}\right\}$ is any sequence such that $t_{k} \rightarrow \tau_{\phi}^{+}$and $\left\{\phi\left(t_{k}\right)\right\}$ converges to a point in $P$ as $k \rightarrow+\infty$, then $V\left(\phi\left(t_{k}\right), t_{k}\right) \rightarrow v_{\phi}$ as $k \rightarrow+\infty$.

Proof. It is well known that $\Omega_{\phi} \cap P \neq \varnothing$ implies that $\tau_{\phi}^{+}=+\infty$; see [1]. Let $\left\{t_{k}\right\}$ be such that $\phi\left(t_{k}\right) \rightarrow p \in P$ and $t_{k} \rightarrow+\infty$ as $k \rightarrow+\infty$ and let $\left\{s_{m}\right\}$ be such that $\phi\left(s_{m}\right) \rightarrow q \in P$ and $s_{m} \rightarrow+\infty$ as $m \rightarrow+\infty$. Then $p, q \in Q$ and the sequences $\left\{V\left(\phi\left(t_{k}\right), t_{k}\right)\right\}$ and $\left\{V\left(\phi\left(s_{m}\right), s_{m}\right)\right\}$ are nonincreasing for all sufficiently large values of the indices. They are bounded below since $V$ is a gauge function. Denote the respective limits by $\tau$ and $\sigma$ and extract subsequences $\left\{t_{k_{i}}\right\}$ and $\left\{s_{m_{i}}\right\}$ from $\left\{t_{k}\right\}$ and $\left\{s_{m}\right\}$ in such a way that $t_{k_{i}} \leqq$ $s_{m_{i}} \leqq t_{k_{i+1}}$. Then

$$
V\left(\phi\left(t_{k_{i+1}}\right), t_{k_{i+1}}\right) \leqq V\left(\phi\left(s_{m_{i}}\right), s_{m_{i}}\right) \leqq V\left(\phi\left(t_{k_{i}}\right), t_{k_{i}}\right)
$$

for all sufficiently large $i$ and, passing to the limit, we have $\tau \leqq \sigma \leqq \tau$.

THEOREM 2. Let the hypotheses of Theorem 1 hold. In addition, suppose that $\lim _{t \rightarrow+\infty} V(x, t)=\lambda(x)$ exists for every $x \in Q$ and that $V$ is continuous at each $x \in Q$, uniformly with respect to $t$ in $I$. Then, if $p \in \Omega_{\phi}, V(p, t) \rightarrow v_{\phi}$ as $t \rightarrow+\infty$.

Proof. Choose any sequence $\left\{t_{k}\right\}$ such that $\phi\left(t_{k}\right) \rightarrow p$ and $t_{k} \rightarrow+\infty$ as $k \rightarrow+\infty$ and let $\varepsilon>0$ be given. There are numbers $T$ and $\delta$ such that 
$|V(p, t)-\lambda(p)|<\varepsilon / 2$ for all $t \geqq T$ and, if $|x-p|<\delta$, then $|V(x, t)-V(p, t)|<$ $\varepsilon / 2$ for all $t$ in $I$. Choose $K$ so large that $k \geqq K$ implies $t_{k} \geqq T$ and $\left|\phi\left(t_{k}\right)-p\right|<$ $\delta$. Then $k \geqq K$ also implies that

$$
\left|\lambda(p)-V\left(\phi\left(t_{k}\right), t_{k}\right)\right| \leqq\left|\lambda(p)-V\left(p, t_{k}\right)\right|+\left|V\left(p, t_{k}\right)-V\left(\phi\left(t_{k}\right), t_{k}\right)\right|<\varepsilon .
$$

Thus $\lambda(p)=v_{\phi}$.

COROLlaRy. The limit set $\Omega_{\phi}$ is a subset of the locus of exactly one of the equations $\lim _{t \rightarrow+\infty} V(x, t) \equiv$ constant.

3. An example. Consider the linear second order equation

$$
x^{\prime \prime}+q(t) x^{\prime}+p(t) x=0
$$

where $q$ is nonnegative and continuous and $p$ is absolutely continuous, nonincreasing and bounded below with $\lim _{t \rightarrow+\infty} p(t)=a$.

Any solution $x=\phi(t), y=\phi^{\prime}(t)$ of $(\mathrm{L})$ will have for its $\omega$-limit set a subset, possibly empty, of the locus of an equation $y^{2}+a x^{2} \equiv$ constant. This is immediately established with the aid of the gauge function $V(x, y, t)=$ $y^{2}+p(t) x^{2}$ and the corollary to Theorem 2 since $D V(x, y, t)=-2 q(t) y^{2}+$ $p^{\prime}(t) x^{2}$.

If $a \leqq 0$, every $\omega$-limit set is a subset of a hyperbola or a pair of lines in the $x y$-plane.

Suppose that $a>0$ and let

$$
x=\phi(t), \quad y=\phi^{\prime}(t)
$$

denote an arbitrary solution of (L). We assert that $(\mathrm{T})$ approaches precisely one (possibly degenerate) ellipse $y^{2}+a x^{2}=c \geqq 0$.

To prove the assertion, assume first, for contradiction, that the $\omega$-limit set $\Omega_{\phi}$ of (T) is empty. The $|\phi(t)|+\left|\phi^{\prime}(t)\right| \rightarrow+\infty$ as $t \rightarrow+\infty$ for, otherwise, $\left(\phi(t), \phi^{\prime}(t)\right)$ would be interior to some ball $x^{2}+y^{2}<r^{2}$ for an unbounded sequence of times and the Bolzano-Weierstrass theorem would imply that $\Omega_{\phi}$ is not empty. Choose an $M>0$ such that $\left(\phi(t), \phi^{\prime}(t)\right)$ is exterior to the ellipse $x^{2}+a y^{2}=M$ for all sufficiently large $t$. Then

$$
V\left(\phi(t), \phi^{\prime}(t), t\right) \geqq\left[\phi^{\prime}(t)\right]^{2}+a \phi^{2}(t) \geqq M
$$

for such $t$ and, since $V\left(\phi(t), \phi^{\prime}(t), t\right)$ is nonincreasing, it has a limit $b$ as $t \rightarrow+\infty$. It follows that $\left[\phi^{\prime}(t)\right]^{2}+a \phi^{2}(t) \leqq b+1$ for all sufficiently large $t$, consequently (T) is bounded as $t \rightarrow+\infty$, a contradiction. Thus $\Omega_{\phi}$ is not empty. 
By the corollary to Theorem $2, \Omega_{\phi}$ lies in precisely one ellipse $y^{2}+a x^{2}=$ $c \geqq 0$. The solution (T) must approach the ellipse as $t \rightarrow+\infty$ because $\Omega_{\phi}$ would be disconnected [2] if (T) did not.

\section{REFERENCES}

1. T. Yoshizawa, Stability theory by Liapunov's second method, Publ. Math. Soc. Japan, no. 9, Math. Soc. Japan, Tokyo, 1966, p. 52. MR 34 \#7896.

2. H. K. Wilson, Ordinary differential equations, Addison-Wesley, Reading, Mass., 1971, pp. 280-282. MR 43 \#6483.

Department of Mathematical Studies, Southern Illinois University, EdwardsVILLE, ILLINOIS 62025 